\title{
Visualization of Design Knowledge Component Relationships to Facilitate Reuse
}

\author{
Shahtab Wahid, Jamie L. Smith, Brandon Berry, C. M. Chewar' ${ }^{1}$ D. Scott McCrickard \\ Center for Human-Computer Interaction and Department of Computer Science \\ Virginia Polytechnic Institute and State University \\ Blacksburg, VA 24061-0106 USA \\ \{swahid, jls05, brberry, cchewar, mccricks\}@vt.edu
}

\begin{abstract}
Within the software development process, reuse at the requirements level has become an increasingly more compelling notion. Following a human-centric approach, this work focuses on both requirements and design solution reuse using a design knowledge repository. In recent years, many improvements have been made to increase reuse through design knowledge repositories, but retrieval of knowledge in the context of design activities continues to be a formidable challenge. We propose a new system, called CERVi, to browse a repository through visualization by exploiting relationships between units of knowledge (in our case, claims). These relationships are key to finding the most appropriate reusable knowledge based on design conditions. Testing shows that CERVi enhances the design knowledge selection process and helps users proceed through structured design decision making. Our approach will be most useful to those interested in unlocking the potential of design knowledge reuse.
\end{abstract}

Keywords: design knowledge reuse, claims, information visualization, human computer interaction

\section{Introduction}

To better understand and manage human-computer interaction (HCI) concepts and principles in software development, designers must focus on relevant artifact features and explicit design tradeoffs. A knowledge schema created specifically for human centric design, a claim is a reusable piece of design knowledge that defines a feature of a specific artifact and a set of impacts on users in incorporating that feature into a system design [1][2][13]. Delivered in informal natural language, claims can cover a wide range of design problems and principles by making explicit the upside and downside tradeoffs (Figure 1) implicitly narrated in a user task scenario [3]. In addition to the design tradeoffs, a claim may also include a list of effects (desired and measurable goals that the implemented design feature should achieve), dependencies (assumptions made by designers that are

${ }^{1}$ Author's current address: Department of Electrical Engineering and Computer

Science, United States Military Academy, West Point, NY 10996 necessary to achieve the proposed effects), issues (design questions generated by the claim), and pointers to related claims [13].

Clicking on monitored information that is displayed in a notification system...

+ can give the user access to more information about the notification

+ may allow completion of their secondary task

- BUT may not provide the expected information

- BUT requires a shift from their primary task

Figure 1. Claim about clicking to access information.

In recent years, research within HCI has recognized that to facilitate reuse, claims must be generalized, classified, stored in a design knowledge repository, and retrieved as appropriate for use within a new design context [1][2][3][12][13]. Within this literature, approaches for repositories of claims (referred to as claims catalogs or claims libraries) have been introduced, although core architecture and feature design are topics of much contention. Although our work extends the concept of a claims library, we feel the general approach can also apply to other forms of design knowledge repositories, to include collections of patterns or scenarios.

The claims library we are specifically interested in [11] stores and indexes claims about notification systems - applications which notify users of monitored information by allocating attention between primary and secondary tasks and providing access to further information. Depending on the usage context of a particular notification system, users desire different levels of interruption, reaction, and comprehension (IRC)-the three critical parameters that classify notification system design artifacts using quantitative values [9].

The purpose of the claims library is to provide meaningful design knowledge and encourage designers to search for and consider claims about systems similar to an application they are currently designing. Each claim in the library is situated in an abstract, four-dimensional space decided by its primary task, notification tasks, IRC values, and design abstraction. The primary task describes a broad user goal, while the notification tasks define user goals at a lower level. The design abstraction refers to an 
artifact and consists of a set of keywords generalizing an interaction between the system and the user. The IRC ratings, presented on a scale from zero to one, represent the impact of the artifact on the user [11].

Unfortunately, as with most knowledge management systems, acquisition is the bottleneck [14]. Component selection is a very important characteristic of reuse [6][8]; however, the current state of the retrieval mechanisms in knowledge repositories today is inadequate. Searches for components are limited to keywords, and classification schemes, such as tasks and IRC values in our case, only serve as parameters to enhance retrieval. Browsing capabilities to navigate from one component to another are nonexistent or also inadequate in such systems. Furthermore, most design knowledge repositories do not support an outlined search strategy, a series of steps they can follow depending on their needs, to ensure that they will find all of the components they need.

A new approach to browsing involves the creation of a networked structure of relationships among claims. This paper proposes the use of claim relationships to enhance the acquisition and selection process of reuse within the context of the claims library. By studying relationships types among knowledge components users can begin to follow links to find more components. Thus, designers can first use components discovered through a traditional search query to find more by browsing and following relationship links based on a certain design need. We seek to establish a goal-oriented search strategy based on the use of particular relationship types under certain search conditions that will enable designers to create more structured designs.

\section{Related work}

Sutcliffe [12][13] asserts that for claims to be an effective component of reuse, they need to be classified and organized in a library. He also touches upon the need for claims relationships within such a library. In a similar vein, Zimmer [16] attempts to apply relationships to design patterns, another form of design knowledge prevalent within the software engineering community. Zimmer categorizes his relationships into three types: a pattern using another pattern, a pattern that is similar to another pattern, and a pattern that is combined with another pattern. While the relationships are useful, they are not expansive enough to cover the various activities of designing.

Libraries that exploit relationships are nothing new. Chen [4] introduces an entity-relationship model for databases. Embly [7] demonstrates a library containing abstract data types (ADT) for reuse that is structured to use relationships among entities. The objective is to locate ADTs in the library and use them in software that is under development. The structures of the relationships facilitate locating, browsing, and building related activities, thus providing increased flexibility. Unfortunately, the nature of the relationship types do not necessarily allow for a goal-oriented search strategy.

Creech et. al. [5] explore the use of hypertext in selecting reuse components. Their belief is that the appropriate use of hypertext to structure components would aid the selection process. The lack of relationship types, however, does not allow for the links to be classified.

Together, these works show that relationships are crucial for creating effective and usable libraries of reusable information.

\section{Claim relationships}

A system interface is the manifestation of a network of claims. Each claim evolves over time and maintains relationships with other claims [15]. Claims Exploration of Relationships Visualization (CERVi) is a tool created to improve system design and knowledge acquisition through the use of claim relationships (Figure 2).

The tool allows designers to find claims by navigating through a visualization of the networked structure of reusable claims. Users can find appropriate claims by analyzing related claims, providing recommendations based on the context of the visualized claim. The tool was designed to enhance the browsing experience and promote cross-platform reuse.

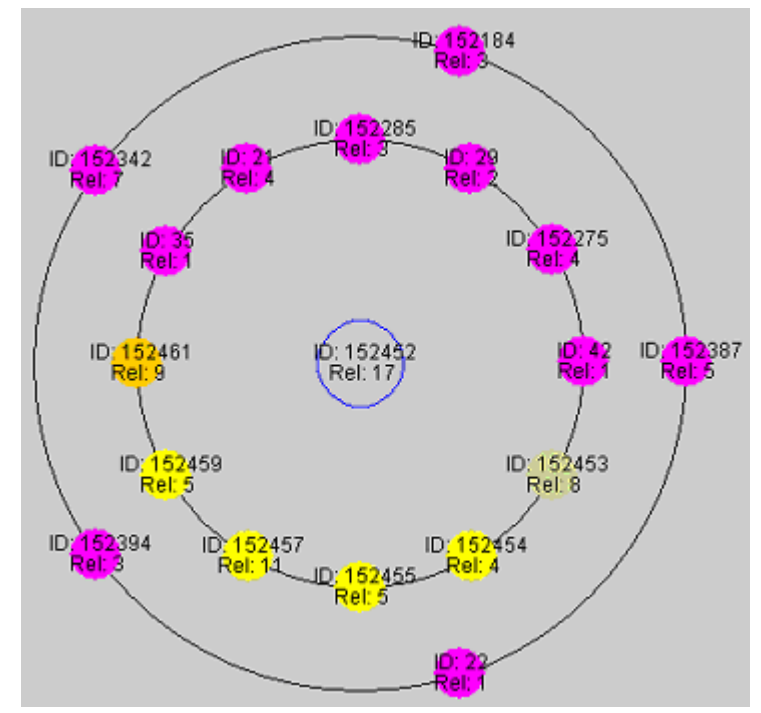

Figure 2. CERVi showing claims related to the center claim by using colors to represent relationship types. The user can also filter based on relationships and see claim details by clicking on a claim.

The relationships embodied in the tool and the work presented in this paper are based on six relationship types that were identified as key to the evolution of claims [15]. Each relationship has a specific purpose in the design process, allowing designers to use the relationships according to their needs. This section defines and explains each relationship that was used in the claims library. 


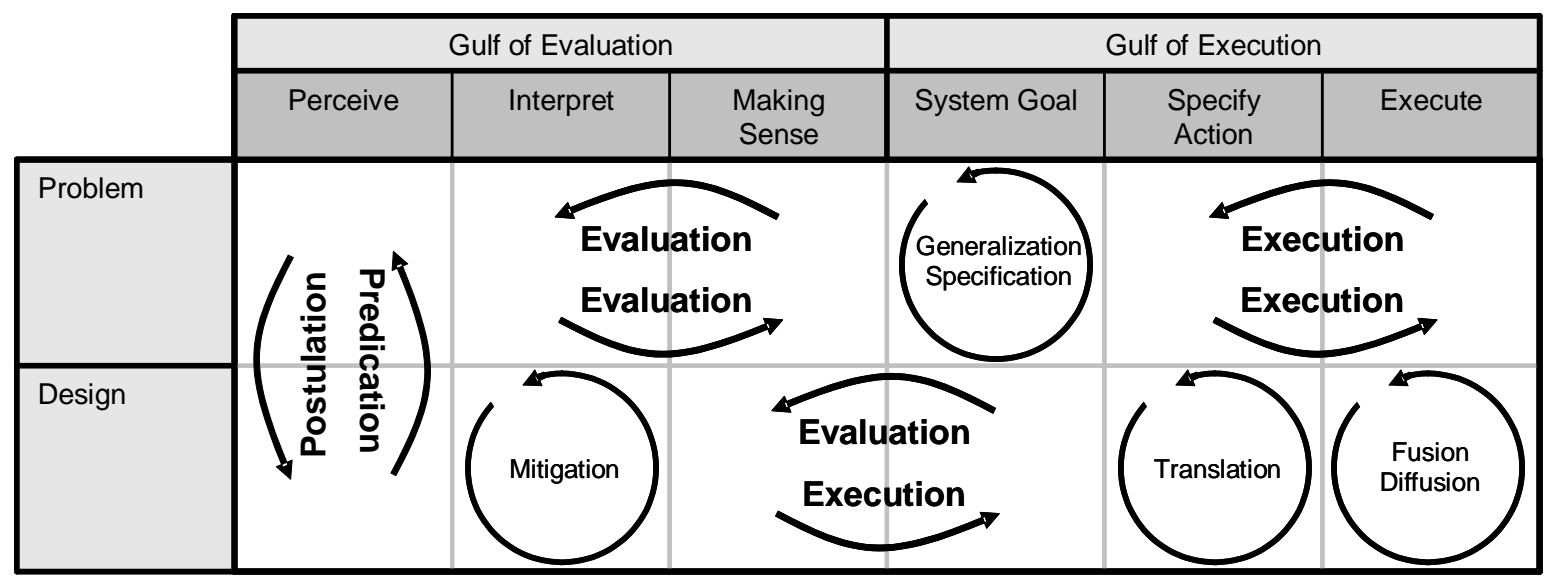

Figure 3. A search strategy for reusable design knowledge, as supported by CERVi.

A postulation relationship leads from a claim about the problem situation (a problem claim) to a claim about a proposed design solution (a design claim), while a predication relationship leads from a design claim to associated problem claims. For example, a problem claim stating that users must be notified of information they are monitoring could lead to a postulating claim about using popup windows to inform users of certain information. By examining a claim's postulation or predication relationships, a designer can easily distinguish a problem claim from a design claim.

Using the postulation relationship, a designer can identify all possible design solutions for a given problem situation. However, while searching the claims library, a designer may not necessarily find the problem claim first. The predication relationship will guide the designer from a reusable design claim to the problem situation, allowing for further exploration of alternative solutions.

Norman argues that designers must allow users to proceed through six stages of action with interface designs to traverse a Gulf of Execution and Gulf of Evaluation [10]. For instance, if a user finds a claim about a feature intended to address Gulf of Execution concerns, s/he can either follow an execution relationship to find another claim in the same Gulf or follow an evaluation relationship to locate a claim related to the Gulf of Evaluation. A claim about identifying an object would have an execution relationship with a claim about clicking on an object to access information. An evaluation relationship would exist between the two claims in the opposite direction.

The generalization relationship links one specific claim to another claim that covers a broader concept. In contrast, the specification relationship links a general claim to one that covers a more focused concept. For example, a claim about using color to convey information has a specification relationship with a claim about using the color red to convey urgent information.

The translation relationship links two similar claims that belong to dissimilar problem domains.
It is common to take two different concepts and incorporate them together in one design. In this way, designers can integrate claims to create new and innovative design solutions. The fusion/diffusion relationship links/decouples two or more claims to one high-level, combination claim.

The mitigation relationship links a claim with a given downside to any claims that provide a solution to that downside. For example, a claim about finding figures of interest has the downside that the user may not notice the figure of interest. A mitigation relationship, however, would solve the problem by pointing to a claim about highlighting figures of interest.

\section{Project highlights}

Through this work, we explored three main concerns: the impact of our tool on a structured design process, the use of a search strategy to promote cross-domain reuse, and the validation of our component selection mechanism within a design knowledge repository.

During the design process, designers following a scenario-based design approach [3] typically locate relevant problem and design claims for each of Norman's stages of action [10] to create a well-planned design. We expect that, by using CERVi to display claims and their relationships, designers will identify problem and design claims and place them correctly within the Gulf of Execution or the Gulf of Evaluation. Successful location and placement of relevant claims will allow even novice designers to better understand the formal process of design, potentially resulting in improved overall designs.

The postulation/predication and execution/evaluation relationships form the basis of a search strategy. They are referred to as the high level relationships because they enable designers to navigate between problem and design domains and between the two Gulfs. The other relationships are called the low level relationships. Instead of allowing for navigation, these relationships allow the designer to focus within a certain area of the design to find more relevant claims. The overall strategy for 
searching supported by CERVi is first to use the high level relationships to find claims that may fit into the overall design and then to use the low level relationships to find the most appropriate claims (Figure 3).

The ability to locate, compare, and select stored components is vital to the success of a software reuse repository. To provide for selection, the claims library must instantiate a strong classification schema and an effective retrieval mechanism based on that classification. Furthermore, the tool's selection mechanism must expose any information the user needs to understand, compare, and use the claims they find [8].

\section{User testing and results}

Emerging from the project highlights, our user testing goals were to determine the impact of CERVi on finding reusable knowledge for interface design, to verify that the defined relationships can be incorporated into a strategy for locating claims, and to validate the tool as a selection mechanism to facilitate claims reuse.

\subsection{Testing procedure}

To accomplish these goals, we developed a design table structured around the problem and design domains and the Gulfs of Execution and Evaluation (Figure 4). We also provided additional information, such as notification tasks and IRC values for each stage of action, in the table. Several claim ID numbers were included under various stages to initiate a search for claims, while other portions of the table were left blank.

We asked sixteen undergraduate HCI students to read a given problem scenario about the need for a notification system and use CERVi and/or the traditional claims library search mechanism in any combination to locate at least ten claims that were relevant to the design they envisioned. The participants were given written definitions of the relationship types, which they could refer back to throughout the design task.

After filling in the design table, participants were asked to complete a survey. Questions were designed to determine the participants' perceived understanding of the relationships and their use, the incorporation of those relationships into participants' search strategies, and the impact of the relationships on the resulting design work.

\subsection{Survey results}

The first section of the survey asked for a brief description of the application the participant envisioned while designing, along with a description of the participant's strategy in searching for claims. Descriptions of the envisioned systems were consistent and generally appropriate for the given scenario. Search strategies were also somewhat consistent. Although some participants did search for claims using the traditional library search mechanism, most participants located the majority of their claims using CERVi due to the ease. They indicated that the postulation/predication and execution/evaluation relationships were mainly used to locate claims across the design table, pointing out the importance of these specific design activities and, consequently, the higher level relationships. Some participants then used the lower level relationships to focus on a specific section of the design table.

The second section of the survey asked participants to rank their understanding of each relationship type on a five point scale, ranging from 'strongly disagree' to 'strongly agree.' The results of these questions show that the participants gained a basic understanding of the four high level relationships (with an average response of 'agree'), while that understanding decreased significantly for the lower level relationships (with an average response of 'neutral' to 'disagree').

The final section of the survey asked questions designed to validate CERVi as a selection mechanism, based on Krueger's [8] definition of selection and its application to reuse. The questions address three key concerns of selection-classification, retrieval, and exposition-as well as selection in general. Responses to the classification questions, related to the conceptual structure of the relationships, indicate a weak understanding of the distinction between high and low level relationships and uncertainty in using those relationships to place claims correctly within the design table. Responses to the retrieval questions, related to the application design, show that participants could easily retrieve a claim based on a displayed relationship. Answers to the exposition questions show uncertainty in using the relationships to understand the underlying purpose of a related claim, but indicate that examination of claim details was a key aspect of the selection process.

\subsection{Design table results}

In addition to the survey results, we also analyzed the design tables in terms of the applicability of the selected claims to the problem scenario and the placement of the claims within the table. We determined a solution set that laid out the correct position for all applicable claims to assess the submitted designs. We compared each participant's design table to the set by counting the number of correct claims, claims incorrectly listed in the problem or design category, claims placed in the wrong Gulf, and claims placed in the correct Gulf, but in the wrong stage. Based on these numbers, each participant was given a design score. Results show that approximately one third of the participants produced effective designs; however, designs created using CERVi were considerably better than those created using the traditional library search mechanism due to the selection of claims that were more applicable to the given scenario. Users who stated that they primarily used CERVi to search for claims committed fewer errors and received higher design scores. Five of those fifteen participants had 


\begin{tabular}{|c|c|c|c|c|c|c|}
\hline & \multicolumn{3}{|c|}{ Gulf of Evaluation } & \multicolumn{3}{|c|}{ Gulf of Execution } \\
\hline & Perceive & Interpret & $\begin{array}{l}\text { Making } \\
\text { Sense }\end{array}$ & System Goal & $\begin{array}{l}\text { Specify } \\
\text { Action }\end{array}$ & Execute \\
\hline Problem & $\begin{array}{l}152452 \\
152456\end{array}$ & & 152389 & 152461 & & 152465 \\
\hline Design & 152184 & 152458 & $\begin{array}{l}152336 \\
152335\end{array}$ & 152362 & & \\
\hline $\begin{array}{l}\text { Notification } \\
\text { Tasks }\end{array}$ & $\begin{array}{l}\text { Evaluate } \\
\text { Identify }\end{array}$ & $\begin{array}{l}\text { Associate } \\
\text { Compare } \\
\text { Evaluate } \\
\text { Interpret }\end{array}$ & $\begin{array}{l}\text { Disassemble } \\
\text { Assemble } \\
\text { Associate } \\
\text { Classify } \\
\text { Compare } \\
\text { Model } \\
\text { Sort } \\
\text { Test }\end{array}$ & $\begin{array}{l}\text { Disassemble } \\
\text { Classify } \\
\text { Decide } \\
\text { Sort }\end{array}$ & $\begin{array}{l}\text { Disassemble } \\
\text { Assemble } \\
\text { Locate } \\
\text { Orient } \\
\text { Plan }\end{array}$ & $\begin{array}{l}\text { Communicate } \\
\text { Explain } \\
\text { Locate } \\
\text { Monitor } \\
\text { Record } \\
\text { Search } \\
\text { Select } \\
\text { Test }\end{array}$ \\
\hline IRC & Lo/Hi/Lo & Lo/Hi/Lo & Lo/Hi/Lo & Lo/Hi/Lo & Lo/Hi/Lo & Lo/Hi/Lo \\
\hline
\end{tabular}

Figure 4. The design table given to the participants, including Norman's stages of action [10].

high design scores that were all the result of primarily using CERVi.

\subsection{Expert user results}

Based on the results of the first user group, we asked a second group of six expert users to perform the same tasks. Before beginning the test, these participants were given a detailed explanation of the relationship types, along with an example of their intended use. This led to everyone using CERVi more than the regular search to find for claims.

Design scores for the second group rose considerably, with five out of six participants creating solid designs with high design scores and scoring higher than the average score of the first user group. Survey results show consistent descriptions of envisioned systems and indicate that the second group of participants had a stronger understanding of the relationships and were more inclined to use them in locating claims. Participants agreed that they understood the distinction between high and low level relationships and could use those relationships to aid in the placement of claims within the design table. Agreement remained high in terms of ease of retrieval and rose in terms of using the relationships to better understand a claim's purpose.

\section{Discussion}

With results of the second user group taken into account, the overall results with respect to our three main concerns are very encouraging. Participants produced considerably better designs by incorporating the relationships into their search strategy. The design scores reflect the completeness and applicability of the submitted designs. Our work is based on the assumption that a design that is complete and applicable is "better" than one that is not. As a result, we can conclude that our selection process is a very promising solution to the known challenges of design knowledge retrieval.

However, there is a need for further investigation in two key areas of future work. Participants in both user groups indicated that they did not use the low level relationships as often as they used the high level relationships. The primary reasons for not using the low level relationships were a lack of understanding, a lack of applicable claims that had lower level relationships, and a belief that the high level relationships were sufficient for retrieving the required ten claims. To continue our studies on the low level relationships, we must either restrict the design table to force the participants to focus on a particular part of the design table or ask users to find more than ten claims. Making this decision will require careful consideration about both the real design process and effective pedagogy for design.

Results improved significantly with the second user group. However, further investigation is still needed, as we do not yet fully understand the impact of providing participants with a full explanation of the relationships prior to completing the design task. Consequently, a third user group, consisting of typical user participants, must be tested after having received a full explanation of the relationships and their intended use. Since our initial testing was controlled in a short lab-based session, this third test should challenge designers with tasks that span several days and are conducted in more ecologically valid conditions.

\section{Conclusions}

Component selection is a vital aspect of successful design knowledge and software reuse. Although the claims library contains an abundance of reusable design knowledge, that knowledge is useless unless it can be 
properly classified, stored, and retrieved for use within a new design context. CERVi brings a number of improvements to the process of component selection, including that it:

- Provides easier browsing capabilities using relationships between design knowledge units - Facilitates identification of claims suitable for a certain location in the design table

- Promotes better designs due to a thorough process of reasoning about psychological effects of task flow

- Enables use of a search strategy to ensure complete designs through task coverage

CERVi shows potential as a successful tool with a positive impact on design. Furthermore, the relationship types embodied by this tool can be successfully incorporated into a search strategy that further aides in retrieving relevant claims. Based on initial results and anticipated results of future development, CERVi is a promising and attested selection mechanism to facilitate reuse of design knowledge. Although our effort lends new hope to the prospect for design knowledge repositories, we are eager to extend our efforts within the Information Reuse and Integration community.

\section{References}

[1] Carroll, J. M. Making use: a design representation. Communications of the ACM Vol. 37, Issue 12, pp. 28-35, December 1994.

[2] Carroll, J. M. and Kellogg, W. A. Artifact as TheoryNexus: Hermeneutics Meets Theory-Based Design. In Proceedings of the Conference on Human Factors in Computing Systems (CHI '89), pp. 7-14, ACM, New York, 1989.

[3] Carroll, J. M. and Rosson, M. B. Getting Around the Task-Artifact Cycle: How to Make Claims and Design by Scenario. ACM Transactions on Information Systems (TOIS), Vol. 10, No. 2, pp. 181212, April 1992.

[4] Chen, P. P. S. The Entity-Relationship Model Towards a Unified View of Data. ACM Transactions of Database Systems (TODS). Vol. 1, Issue 1, pp 936, 1976.

[5] Creech, M. L., Freeze, D. F., Griss, M. L. Using Hypertext in Selecting Reusable Software Components. In Proceedings of $3^{\text {rd }}$ Annual ACM Conference on Hypertext and Hypermedia. San Antonio, TX, pp. 25-38, 1991.
[6] Dusink, L. and van Katwijk, J. Reuse Dimensions. In Proceedings of Symposium on Software Reusability, pp. 137-149, Seattle, WA, 1995.

[7] Embley, D. W. and Woodfield, S. N. A Knowledge Structure for Reusing Abstract Data Types. In Proceedings of $9^{\text {th }}$ International Conference on Software Engineering, Monterey, CA, pp. 360-368, 1987.

[8] Krueger, C. W. Software Reuse, ACM Computing Surveys (CSUR) Vol. 24, Issue 2, pp. 131-183, June 1992.

[9] McCrickard, D. Scott, Chewar, C. M., Somervell, J. P., and Ndiwalana, A. A Model for Notification Systems Evaluation--Assessing User Goals for Multitasking Activity. ACM Transactions on Computer-Human Interaction, Vol. 10, No. 4, pp. 312-338, December 2003.

[10] Norman, D. A. Cognitive Engineering. In D. A. Norman \& S. W. Draper, Eds. User Centered Design, pp. 31-62, Hillsdale, NJ: Erlbaum, 1986.

[11] Payne, C., Allgood, C. F., Chewar, C. M., Holbrook, C., and McCrickard, D. S. Generalizing Interface Design Knowledge: Lessons Learned from Developing a Claims Library. IEEE International Conference on Information Reuse and Integration (IRI 2003), pp. 362-369, Las Vegas, October 27-29, 2003.

[12] Sutcliffe, A. G. On the Effective Use and Reuse of HCI Knowledge. ACM Transactions on ComputerHuman Interaction (TOCHI), Vol. 7, Issue 2, pp. 197-221, June 2000.

[13] Sutcliffe, A. G. and Carroll, J. M. Designing Claims for Reuse in Interactive Systems Design. International Journal of Human-Computer Studies, Vol. 50, No. 3, pp. 213-241, 1999.

[14] Wagner, W. P. Issues in Knowledge Acquisition. In Proceedings of ACM SIGBDP Conference on Trends and Directions in Expert Systems, pp. 247-261, Orlando, FL, 1990.

[15] Wahid, S. Allgood, C. F., Chewar, C. M., McCrickard, D. S. Entering the Heart of Design: Relationships for Tracing Claims Evolution. Conference on Software Engineering and Knowledge Engineering (SEKE 2004). pp. 167-172, Banff, Alberta, CA, June 21-24, 2004.

[16]Zimmer, W. Relationships between Design Patterns. In J. O. Coplien and D. C. Schmidt, editors, Pattern Languages of Program Design, pp. 354-364, Addison-Wesley, 1995. 